\title{
THE ABSOLUTE EULER SUMMABILITY OF FOURIER SERIES
}

\author{
B. KWEE
}

(Received 24 October 1969)

Communicated by E. Strzelecki

\section{Introduction}

The series $\sum_{n=0}^{\infty} a_{n}$ is said to be summable $\left|E_{\alpha}\right|(0<\alpha<1)$ if

$$
t_{n}=\sum_{v=0}^{n}\left(\begin{array}{l}
n \\
v
\end{array}\right) \alpha^{v}(1-\alpha)^{n-v} s_{v},
$$

where $s_{v}=a_{0}+a_{1}+\cdots+a_{v}$, and

$$
\sum_{n=1}^{\infty}\left|t_{n}-t_{n-1}\right|<\infty
$$

Since

$$
\tau_{n}=\sum_{v=1}^{n}\left(\begin{array}{l}
n \\
v
\end{array}\right) \alpha^{v}(1-\alpha)^{n-v} v a_{v}=n\left(t_{n}-t_{n-1}\right)
$$

(see [2]), (1) is equivalent to

$$
\sum_{n=1}^{\infty}\left|\frac{\tau_{n}}{n}\right|<\infty
$$

We suppose throughout that $f(x)$ is a periodic function with period $2 \pi$, integrable in the Lebesgue sense. Let

(3)

$$
\begin{aligned}
f(x) & \sim \frac{a_{0}}{2}+\sum_{n=1}^{\infty}\left(a_{n} \cos n x+b_{n} \sin n x\right) \\
& =\sum_{n=0}^{\infty} A_{n}(x) .
\end{aligned}
$$

The series conjugate to (3) is

$$
\sum_{n=1}^{\infty}\left(b_{n} \cos n x-a_{n} \sin n x\right)=\sum_{n=1}^{\infty} B_{n}(x)
$$

and the differentiated series of (3) is

$$
\sum_{n=1}^{\infty} n B_{n}(x)
$$


We write

$$
\begin{aligned}
& \phi(t)=\phi_{x}(t)=\frac{1}{2}\{f(x+t)+f(x-t)-2 s\}, \\
& \psi(t)=\frac{1}{2}\{f(x+t)-f(x-t)\} .
\end{aligned}
$$

N. Tripathy [3] has shown the condition that $\phi(t)$ is of bounded variation in $(0, \pi)$ does not ensure the summability of $(3)$ by $\left|E_{\alpha}\right|$.

In this paper we shall prove

THEOREM 1. If $g(t)=\phi(t) \log 1 / t$ is of bounded variation in $0 \leqq t \leqq \delta<1$, then $\sum_{n=0}^{\infty} A_{n}(x)$ is summable $\left|E_{\alpha}\right| . g(t)$ cannot be replaced by $g_{\eta}(t)=\phi(t)(\log 1 / t)^{n}$ for $0<\eta<1$.

THEOREM 2. If

$$
\int_{0}^{\delta} \log \frac{1}{u}|d \phi(u)|<\infty,
$$

then $\sum_{n=0}^{\infty} A_{n}(x)$ is summable $\left|E_{\alpha}\right|$.

J. M. Whittaker [4] proved that, if $\phi(t) / t \in L(0, \delta)$, then the Fourier series (3) is summable $|A|$. We shall prove

THEOREM 3. The condition $\phi(t) / t^{\eta} \in L(0, \pi)$, where $\eta<2$, does not ensure that (3) is summable $\left|E_{\alpha}\right|$.

However we have

THEOREM 4. If $\phi(t) / t^{2} \in L(0, \delta)$, then (3) is summable $\left|E_{\alpha}\right|$.

For the conjugate series we have

THEOREM 5. If $\psi(+0)=0$ and

$$
\int_{0}^{\delta} \log \frac{1}{t}|d \psi(t)|<\infty
$$

then the conjugate series (4) is summable $\left|E_{\alpha}\right|$.

Finally we shall prove the following theorem on the differentiated series.

THEOREM 6. If $\psi(+0)=0$ and

$$
\int_{0}^{\delta} \frac{1}{u^{2}}|d \psi(u)|<\infty,
$$

then (5) is summable $\left|E_{\alpha}\right| .(6)$ cannot be replaced by

$$
\int_{0}^{\delta} \frac{1}{u^{\eta}}|d \psi(u)|<\infty
$$

for any $\eta<2$. 


\section{Proof of Theorem 1}

Let

$$
G_{n}(u)=\sum_{v=1}^{n}\left(\begin{array}{l}
n \\
v
\end{array}\right) \alpha^{v}(1-\alpha)^{n-v} v \cos v u
$$

Then

$$
\begin{aligned}
\tau_{n} & =\frac{2}{\pi} \int_{0}^{\pi} \phi(u) G_{n}(u) d u \\
& =\frac{2}{\pi}\left(\int_{0}^{\delta}+\int_{\delta}^{\pi}\right) \phi(u) G_{n}(u) d u \\
& =\frac{2}{\pi}\left(I_{n}^{\prime}+I_{n}^{\prime \prime}\right) .
\end{aligned}
$$

Now

(9)

$$
\begin{aligned}
G_{n}(u) & =\frac{d}{d u}\left(\sum_{\nu=1}^{n}\left(\begin{array}{l}
n \\
v
\end{array}\right) \alpha^{v}(1-\alpha) \sin v u\right) \\
& =\operatorname{Im} \frac{d}{d u}\left(1-\alpha+\alpha e^{i u}\right)^{n} \\
& =\operatorname{Im}\left\{n \alpha i e^{i u}\left(1-\alpha+\alpha e^{i u}\right)^{n-1}\right\} \\
& =n \alpha \rho^{n-1}(u) \operatorname{Im}\left\{i e^{i u+i \overline{n-1} \theta(u)}\right\} \\
& =n \alpha \rho^{n-1}(u) \cos (u+\overline{n-1} \theta(u)),
\end{aligned}
$$

where

$$
\begin{aligned}
& \rho(u)=\sqrt{1-4 \alpha(1-\alpha) \sin ^{2} \frac{u}{2}}, \\
& \theta(u)=\tan ^{-1} \frac{\alpha \sin u}{1-\alpha+\alpha \cos u} .
\end{aligned}
$$

It is clear that $\rho(u) \leqq e^{-c u^{2}}(0 \leqq u \leqq \pi)$, where $c$ is a positive constant. Hence

$$
\begin{aligned}
\sum_{n=1}^{\infty} \frac{\left|I_{n}^{\prime \prime}\right|}{n} & =0\left(\int_{\delta}^{\pi}|\phi(u)|\left(\sum_{n=1}^{\infty} \rho^{n-1}(u)\right) d u\right) \\
& =0\left(\sum_{n=1}^{\infty} e^{-c n \delta^{2}}\right) \\
& =0(1) .
\end{aligned}
$$

Let

$$
E_{n}(u)=\int_{0}^{u}\left(\log \frac{1}{t}\right)^{-1} G_{n}(t) d t
$$


Then

$$
\begin{aligned}
I_{n}^{\prime} & =\int_{0}^{\delta} g(u)\left(\log \frac{1}{u}\right)^{-1} G_{n}(u) d u \\
& =g(\delta) E_{n}(\delta)-\int_{0}^{\delta} E_{n}(u) d g(u) .
\end{aligned}
$$

Hence

$$
\sum_{n=1}^{\infty} \frac{\left|I_{n}^{\prime}\right|}{n}<\infty
$$

if

$$
\sum_{n=1}^{\infty} \frac{\left|E_{n}(u)\right|}{n}<\infty
$$

uniformly for $0 \leqq u \leqq \delta$. Let

$$
\begin{aligned}
H_{n}(u) & =\int_{0}^{u} G_{n}(t) d t \\
& =\sum_{v=1}^{n}\left(\begin{array}{l}
n \\
v
\end{array}\right) \alpha^{v}(1-\alpha)^{n-v} \sin v u \\
& =\rho^{n}(u) \sin n \theta(u) .
\end{aligned}
$$

Then

$$
E_{n}(u)=\left(\log \frac{1}{u}\right)^{-1} H_{n}(u)-\int_{0}^{u} \frac{1}{t}\left(\log \frac{1}{t}\right)^{-2} H_{n}(t) d t .
$$

Let $N$ be the smallest positive integer such that $N u^{4}>1$. Then

$$
\begin{aligned}
\left(\log \frac{1}{u}\right)^{-1} \sum_{n=1}^{\infty} \frac{H_{n}(u)}{n} & \geqq\left(\log \frac{1}{u}\right)^{-1}\left(\sum_{n=1}^{N}\left|\frac{\rho^{n}(u) \sin n \theta(u)}{n}\right|+\sum_{n=N+1}^{\infty} \frac{e^{-c n u^{2}}}{n}\right) \\
& =0\left(\left(\log \frac{1}{u}\right)^{-1} \sum_{n=1}^{N} \frac{1}{n}\right)+0\left(\sum_{n=1}^{\infty} \frac{e^{-c \sqrt{ } n}}{n}\right) \\
& =0(1),
\end{aligned}
$$

uniformly for $0<u \leqq \delta$. Now write

$$
\int_{0}^{u} \frac{1}{t}\left(\log \frac{1}{t}\right)^{-2} H_{n}(t) d t=J_{n}^{\prime}+J_{n}^{\prime \prime}
$$

where 


$$
\begin{aligned}
& J_{n}^{\prime}= \begin{cases}\int_{0}^{u} \frac{1}{t}\left(\log \frac{1}{t}\right)^{-2} H_{n}(t) d t & \left(u \leqq \frac{1}{n}\right), \\
\int_{0}^{1 / n} \frac{1}{t}\left(\log \frac{1}{t}\right)^{-2} H_{n}(t) d t & \left(u>\frac{1}{n}\right), \\
0 & \left(u \leqq \frac{1}{n}\right),\end{cases} \\
& J_{n}^{\prime \prime}= \begin{cases}\int_{1 / n}^{u} \frac{1}{t}\left(\log \frac{1}{t}\right)^{-2} H_{n}(t) d t & \left(u>\frac{1}{n}\right) .\end{cases}
\end{aligned}
$$

Since $\sin v t=0(v t)$, we have

$$
\begin{aligned}
H_{n}(t) & =0\left(t \sum_{v=1}^{n}\left(\begin{array}{l}
n \\
v
\end{array}\right) \alpha^{v}(1-\alpha)^{n-v} v\right) \\
& =0(n t) .
\end{aligned}
$$

Hence

$$
\begin{aligned}
\sum_{n=1}^{\infty} \frac{\left|J_{n}^{\prime}\right|}{n} & =0\left(\sum_{n=1}^{\infty} \int_{0}^{1 / n}\left(\log \frac{1}{t}\right)^{-2} d t\right) \\
& =0\left(\sum_{n=2}^{\infty} \frac{1}{n \log ^{2} n}\right) \\
& =0(1) .
\end{aligned}
$$

It is clear that

$$
\sum_{v=1}^{n} \frac{1}{v}\left(\begin{array}{l}
n \\
v
\end{array}\right) \alpha^{v}(1-\alpha)^{n-v}=0\left(\frac{1}{n}\right),
$$

and hence, for $u>1 / n$,

$$
\begin{aligned}
J_{n}^{\prime \prime} & =n(\log n)^{-2} \int_{1 / n}^{\xi_{n}}\left(\sum_{\nu=1}^{n}\left(\begin{array}{l}
n \\
v
\end{array}\right) \alpha^{\nu}(1-\alpha)^{n-v} \sin v t\right) d t \\
& =0\left(\frac{1}{\log ^{2} n}\right)
\end{aligned}
$$

where $1 / n \leqq \xi_{n} \leqq u$. It follows that

$$
\sum_{n=1}^{\infty} \frac{\left|J_{n}^{\prime \prime}\right|}{n}=0(1)
$$

From (13), (14), (15), (16) and (17), we see that (12), and hence (11) holds. The first part of Theorem 1 follows from (8), (10) and (11).

To prove the second part, we required the following lemma due to $\mathrm{L}$. S. Bosanquet and H. Kestleman [1]. 
Lemma 1. Suppose that $f_{n}(x)$ is measurable in $(a, b)$, where $b-a \leqq \infty$, for $n=1,2, \cdots$. Then a necessary and sufficient condition that, for every function $h(x)$ summable over $(a, b)$, the functions $f_{n}(x) h(x)$ should be summable over $(a, b)$ and

$$
\sum_{n=1}^{\infty}\left|\int_{a}^{b} h(x) f_{n}(x) d x\right|<\infty
$$

is that $\sum_{n=1}^{\infty}\left|f_{n}(x)\right|$ should be essentially bounded in $(a, b)$.

(10) is unaffected when $g(t)$ is replaced by $g_{\eta}(t)$. Let

$$
E_{n}^{\eta}(u)=\int_{0}^{u}\left(\log \frac{1}{t}\right)^{-\eta} G_{n}(t) d t
$$

Then

$$
I_{n}^{\prime}=g_{\eta}(\delta) E_{n}^{\eta}(\delta)-\int_{0}^{\delta} E_{n}^{\eta}(u) d g_{\eta}(u)
$$

We have

$$
E_{n}^{\eta}(\delta)=\left(\log \frac{1}{\delta}\right)^{-\eta} H_{n}(\delta)-\eta \int_{0}^{\delta} \frac{1}{t}\left(\log \frac{1}{t}\right)^{-\eta-1} H_{n}(t) d t .
$$

Since

$$
\begin{aligned}
\sum_{n=1}^{\infty} \frac{H_{n}(\delta)}{n} & =0\left(\sum_{n=1}^{\infty} \frac{e^{-c n \delta^{2}}}{n}\right) \\
& =0(1),
\end{aligned}
$$

and (16), (17) remain valid when $(\log 1 / t)^{-2}$ is replaced by $(\log 1 / t)^{-\eta-1}$,

$$
\sum_{n=1}^{\infty} \frac{\left|E_{n}^{\eta}(\delta)\right|}{n}<\infty
$$

It follows from Lemma 1, (18) and (19) that a necessary condition for (11) to hold is that

$$
\sum_{n=1}^{\infty} \frac{\left|E_{n}^{\eta}(u)\right|}{n}
$$

should be essentially bounded for $0 \leqq u \leqq \delta$. Now

$$
E_{\eta}^{\eta}(u)=\left(\log \frac{1}{u}\right)^{-\eta} H_{n}(u)-\eta \int_{0}^{u} \frac{1}{t}\left(\log \frac{1}{t}\right)^{-\eta-1} H_{n}(t) d t,
$$

and from (15), (16) and (17) with $(\log 1 / t)^{-2}$ replaced by $(\log 1 / t)^{-\eta-1}$,

$$
\sum_{n=1}^{\infty} \frac{1}{n}\left|\int_{0}^{u} \frac{1}{t}\left(\log \frac{1}{t}\right)^{-n-1} H_{n}(t) d t\right|<\infty
$$


uniformly for $0 \leqq u \leqq \delta$. Since (see [3], page 24)

$$
\left(\log \frac{1}{u}\right)^{-\eta} \sum_{n=1}^{\infty} \frac{\left|H_{n}(u)\right|}{n} \rightarrow \infty
$$

as $u \rightarrow 0,(20)$ is not essentially bounded. This proves the second part of the theorem.

\section{Proof of Theorem 2}

We shall deduce Theorem 2 from Theorem 1.

Suppose that the conditions of Theorem 2 are satisfied. Then $\phi(u)$ is of bounded variation in $(0, \delta)$, and hence it must tend to a limit as $t \rightarrow 0$. By altering the value of $s$ if necessary, we may suppose that this limit is 0 . (Note that the hypothesis of Theorem 2 is unaffected by a change of the value of $s$.) Now

$$
\int_{0}^{\delta}|d g(u)| \leqq \int_{0}^{\delta} \log \frac{1}{u}|d \phi(u)|+\int_{0}^{\delta} \frac{1}{u}|\phi(u)| d u .
$$

The first term on the right of $(21)$ is finite by hypothesis. Since $\phi(u) \rightarrow 0$ as $u \rightarrow 0$,

$$
|\phi(u)| \leqq \int_{0}^{u}|d \phi(t)|
$$

so that the second term on the right of (21) does not exceed

$$
\begin{aligned}
\int_{0}^{\delta} \frac{1}{u} \int_{0}^{u}|d \phi(t)| d u & =\int_{0}^{\delta}|d \phi(t)| \int_{u}^{\delta} \frac{d t}{t} \\
& =\int_{0}^{\delta} \log \frac{\delta}{u}|d \phi(u)| \\
& <\infty .
\end{aligned}
$$

Hence the result.

\section{Proof of Theorem 3}

Since

$$
\tau_{n}=\frac{2}{\pi} \int_{0}^{\pi} \phi(u) G_{n}(u) d u,
$$

it follows from Lemma 1 that (3) is summable $\left|E_{\alpha}\right|$ if and only if $u^{n} \sum_{n=1}^{\infty}\left|G_{n}(u)\right| / n$, is essentially bounded for $0 \leqq u \leqq \pi$, or, from (9),

$$
u^{n} \sum_{n=1}^{\infty} \rho^{n-1}(u)|\cos (u+\overline{n-1} \theta(u))|
$$


is essentially bounded for $0 \leqq u \leqq \pi$. Let $M=[1 / u]$ and $N=\left[1 / u^{2}\right]$. Then (23) is greater than

$$
\begin{aligned}
u^{\eta} \sum_{n=M}^{N} \rho^{n-1}(u) \mid \cos (u+\overline{n-1} & \theta(u)) \mid \geqq u^{\eta} \sum_{n=M}^{N} \rho^{n-1}(u) \cos ^{2}(u+\overline{n-I} \theta(u)) \\
= & \frac{u^{\eta}}{2} \sum_{n=M}^{N} \rho^{n-1}(u)+\frac{u^{\eta}}{2} \sum_{n=M}^{N} \rho^{n-1}(u) \cos (2 u+\overline{2 n-1} \theta(u)) \\
= & S_{1}+S_{2} .
\end{aligned}
$$

Without loss of generality, we assume that $1<\eta<2$. Then

$$
\begin{aligned}
S_{2} & =0\left\{u^{\eta} \rho^{M-1}(u)\left|\max _{M \leqq m \leqq N} \sum_{n=M}^{m} \cos (2 u+2 \overline{n-1} \theta(u))\right|\right\} \\
& =0\left(\frac{u^{\eta} e^{-c M u^{2}}}{\sin \theta(u)}\right) \\
& =0(1) .
\end{aligned}
$$

There exists a positive constant $c_{1}$ such that $\rho^{2}(u) \geqq e^{-c_{1} u^{2}}$. Hence, for $n \leqq 1 / u^{2}$, $\rho^{n-1}(u) \geqq c_{2}$ for some constant $c_{2}>0$. Therefore

$$
S_{1} \geqq \frac{c_{2}(N-M) u^{\eta}}{2} \rightarrow \infty
$$

as $u \rightarrow 0+$. Hence (23) is not essentially bounded.

\section{Proof of Theorem 4}

It follows from (22) that (3) is summable $\left|E_{\alpha}\right|$ if

$$
u^{2} \sum_{n=1}^{\infty} \frac{1}{n}\left|G_{n}(u)\right|<\infty
$$

uniformly for $0 \leqq u \leqq \pi$. Now the left hand side of (24) is equal to

$$
\begin{aligned}
\alpha u^{2} \sum_{n=1}^{\infty} \rho^{n-1}(u)|\cos (u+\overline{n-1} \theta(u))| & =0\left(u^{2} \sum_{n=1}^{\infty} e^{-c n u^{2}}\right) \\
& =0\left(u^{2} \int_{1}^{\infty} e^{-c u^{2} y} d y\right) \\
& =0(1)
\end{aligned}
$$

uniformly for $0<u<\pi$. Hence (24) is satisfied. 


\section{Proof of Theorem 5}

Let

$$
F_{n}(u)=\sum_{v=1}^{n}\left(\begin{array}{l}
n \\
v
\end{array}\right) \alpha^{v}(1-\alpha)^{n-v} v \sin v u
$$

Then

$$
\begin{aligned}
\tau_{n} & =\sum_{v=1}^{n}\left(\begin{array}{l}
n \\
v
\end{array}\right) \alpha^{v}(1-\alpha)^{n-v} v B_{v}(x) \\
& =\frac{2}{\pi} \int_{0}^{\pi} \psi(u) F_{n}(u) d u \\
& =\frac{2}{\pi}\left(\int_{0}^{\delta}+\int_{\delta}^{\pi}\right) \psi(u) F_{n}(u) d u \\
& =\frac{2}{\pi}\left(X_{n}^{\prime}+X_{n}^{\prime \prime}\right) .
\end{aligned}
$$

We have

$$
F_{n}(u)=n \alpha \rho^{n-1}(u) \sin (u+\overline{n-1} \theta(u)) .
$$

Hence

$$
\begin{aligned}
\sum_{n=1}^{\infty} \frac{\left|X_{n}^{\prime \prime}\right|}{n} & =0\left(\sum_{n=1}^{\infty} e^{-(n-1) c \delta^{2}}\right) \\
& =0(1) .
\end{aligned}
$$

Now

$$
\begin{aligned}
X_{n}^{\prime} & =\left\{-\psi(u) \int_{u}^{\delta} F_{n}(t) d t\right\}_{0}^{\delta}+\int_{0}^{\delta}\left(\int_{u}^{\delta} F_{n}(t) d t\right) d \psi(u) \\
& =\int_{0}^{\delta}\left(\int_{u}^{\delta} F_{n}(t) d t\right) d \psi(u)
\end{aligned}
$$

so that

$$
\sum_{n=1}^{\infty} \frac{\left|X_{n}^{\prime}\right|}{n}<\infty
$$

if

$$
\sum_{n=1}^{\infty} \frac{1}{n}\left|\int_{u}^{\delta} F_{n}(t) d t\right|=0\left(\frac{1}{u}\right)
$$

Since

$$
\int_{u}^{\delta} F_{n}(t) d t=\rho^{n}(u) \cos n \theta(u)-\rho^{n}(\delta) \cos n \theta(\delta),
$$

we have 


$$
\begin{aligned}
\sum_{n=1}^{\infty} \frac{1}{n}\left|\int_{u}^{\delta} F_{n}(t) d t\right| & =0\left(\sum_{n=1}^{\infty} \frac{e^{-n c u^{2}}}{n}\right) \\
& =0\left(\int_{1}^{\infty} \frac{e^{-c u^{2} y}}{y} d y\right) \\
& =0\left(\int_{1}^{1 / u} \frac{e^{-c u^{2} y}}{y} d y\right)+0\left(\int_{1 / u}^{\infty} \frac{e^{-c u^{2} y}}{y} d y\right) \\
& =0\left(u \int_{1}^{1 / u} \frac{d y}{y}\right)+0\left(\int_{1 / u}^{\infty} e^{-c u^{2} y} d y\right) \\
& =0\left(\frac{1}{u}\right) .
\end{aligned}
$$

Hence (27) holds and the theorem follows from (25), (26) and (27).

\section{Proof of Theorem 6}

We have

$$
\begin{aligned}
\tau_{n} & =\sum_{v=1}^{n}\left(\begin{array}{l}
n \\
v
\end{array}\right) \alpha^{v}(1-\alpha)^{n-v} v^{2} B_{n}(x) \\
& =-\frac{2}{\pi} \int_{0}^{\pi} \psi(u) \frac{d}{d u} G_{n}(u) d u \\
& =-\frac{2}{\pi}\left(\int_{0}^{\delta}+\int_{\delta}^{\pi}\right) \psi(u) \frac{d}{d u} G_{n}(u) d u \\
& =-\frac{2}{\pi}\left(Y_{n}^{\prime}+Y_{n}^{\prime \prime}\right) .
\end{aligned}
$$

Since

$$
\begin{aligned}
& \frac{d}{d u} G_{n}(u)=-n(n-1) \alpha^{2} \rho^{n-2}(u) \sin (2 u+\overline{n-2} \theta(u))-n \alpha \rho^{n-1}(u) \sin (u+\overline{n-1} \theta(u)) \\
& =0\left(n^{2} e^{-n c u^{2}}\right), \\
& \sum_{n=1}^{\infty} \frac{\left|Y_{n}^{\prime \prime}\right|}{n}=0\left(\int_{\delta}^{\pi}|\psi(u)|\left(\sum_{n=1}^{\infty} n e^{-n c u^{2}}\right) d u\right) \\
& =0\left(\sum_{n=1}^{\infty} n e^{-n c \delta^{2}}\right) \\
& =0(1) .
\end{aligned}
$$


We have

$$
Y_{n}^{\prime}=\psi(\delta) G_{n}(\delta)-\int_{0}^{\delta} G_{n}(u) d \psi(u)
$$

From (23),

$$
\begin{aligned}
\sum_{n=1}^{\infty} \frac{\left|G_{n}(\delta)\right|}{n} & =0\left(\sum_{n=1}^{\infty} \frac{e^{-n c \delta^{2}}}{n}\right) \\
& =0(1)
\end{aligned}
$$

and

$$
\begin{aligned}
\sum_{n=1}^{\infty} \frac{\left|G_{n}(u)\right|}{n} & =\alpha \sum_{n=1}^{\infty} \rho^{n-1}(u)|\cos (u+\overline{n-1} \theta(u))| \\
& =0\left(\sum_{n=1}^{\infty} e^{-n c u^{2}}\right) \\
& =0\left(\int_{1}^{\infty} e^{-c u^{2} y} d y\right) \\
& =0\left(\frac{1}{u^{2}}\right) .
\end{aligned}
$$

Hence

$$
\sum_{n=1}^{\infty} \frac{\left|Y_{n}^{\prime}\right|}{n}=0(1)
$$

It follows from (28) and (30) that (2) holds, and hence the first part of Theorem 6 is true.

When (6) is replaced by (7), (28) and (29) are not affected. Since, from the proof of Theorem $3, u^{n} \sum_{n=1}^{\infty}\left|G_{n}(u)\right| / n$ is not essentially bounded, there exists a summable function $a(x)$ such that

$$
\sum_{n=1}^{\infty} \frac{1}{n}\left|\int_{0}^{\delta} u^{\eta} a(u) G_{n}(u) d u\right|=\infty
$$

Let $\psi(u)=\int_{0}^{u} u^{\eta} a(u) d u$. Then $\psi(+0)=0$. Since

$$
\begin{aligned}
\left|\tau_{n}\right| & \geqq \frac{2}{\pi}\left|Y_{n}^{\prime}\right|-\frac{2}{\pi}\left|Y_{n}^{\prime \prime}\right| \\
& \geqq \frac{2}{\pi}\left|\int_{0}^{\delta} G_{n}(u) d \psi(u)\right|-\frac{2}{\pi}\left|\psi(\delta) G_{n}(\delta)\right|-\frac{2}{\pi}\left|Y_{n}^{\prime \prime}\right|,
\end{aligned}
$$

we have

$$
\sum_{n=1}^{\infty} \frac{\left|\tau_{n}\right|}{n}=\infty
$$

which proves the second part of the theorem. 


\section{References}

[1] L. S. Bosanquet and H. Kestleman, 'The absolute convergence of a series of integrals', Proc. London Math. Soc. (2), 45 (1939) 88-97.

[2] K. Knopp and G. G. Lorentz, 'Beiträge zur absoluten Limitierung', Arch. Math. 2 (1949-50), 10-16.

[3] N. Tripathy, 'On the absolute Hausdorff summability of Fourier series', Jour. London Math. Soc. 44 (1969), 15-25.

[4] J. M. Whittaker, 'The absolute summability of Fourier series', Proc. Edinburgh Math. Soc. 2 (1930), 1-5.

Department of Mathematics

University of Malaya

Kuala Lumpur

Malaysia 